\title{
COMPOSIÇão Florística da FloReSta CILIAR do Rio MANDASSAIA, Parque Nacional da Chapada Diamantina, Bahia, Brasil
}

\author{
Alexandre Antunes Ribeiro-Filho ${ }^{1}$, Ligia Silveira Funch ${ }^{1,3}$ \\ \& Maria Jesus Nogueira Rodal ${ }^{2}$
}

\begin{abstract}
Resumo
(Composição florística da floresta ciliar do rio Mandassaia, Parque Nacional da Chapada Diamantina, Bahia, Brasil) Realizou-se o levantamento das angiospermas presentes em um trecho de floresta ciliar, adjacente ao rio Mandassaia ( $12^{\circ} 33^{\prime} \mathrm{S}$ e $41^{\circ} 25^{\prime} \mathrm{W}$ ), pertencente à bacia Santo Antônio e situado no município de Lençóis, Bahia. $\mathrm{O}$ objetivo foi conhecer sua flora e verificar o papel da altitude e distância geográfica em sua composição, comparando os resultados com levantamentos de matas ciliares previamente realizados na referida bacia. Foram registradas 116 espécies distribuídas em 96 gêneros e 51 famílias, sendo Melastomataceae, Fabaceae, Orchidaceae, Asteraceae, Myrtaceae, Euphorbiaceae e Apocynaceae, as famílias de maior riqueza. Os resultados da análise de similaridade florística, baseada no componente arbóreo, entre a floresta ciliar do rio Mandassaia e as demais florestas ciliares estudadas na bacia Santo Antônio indicaram que distância geográfica foi o fator mais importante, uma vez que as áreas mais próximas tiveram valores de similaridade semelhantes, independente de sua altitude. Como espécies indicadoras de florestas ciliares da bacia Santo Antônio, na borda oriental da Chapada Diamantina, destacam-se Alchornea triplinervia, Clusia nemorosa, Simarouba amara, Tapirira guianensis e Vochysia pyramidalis. Palavras-chave: florística, Chapada Diamantina, mata ciliar.
\end{abstract}

\section{Abstract}

(The floristics of a gallery forest along the Mandassaia River, Chapada Diamantina National Park, Bahia State, Brazil) A floristic survey of angiosperm species were performed in a gallery forest along the course of the Mandassaia River (an affluent of the Santo Antônio River) in the Municipality of Lençóis, Chapada Diamantina, Bahia State, Brazil $\left(12^{\circ} 33^{\prime} \mathrm{S} \times 41^{\circ} 25^{\prime} \mathrm{W}\right)$. We intended to describe the flora and examine the potential role of altitude and geographical distance on the flora composition, comparing results with previous surveys of gallery forests at different locations at the same major watershed. The floristic survey identified 116 species, belonging to 96 genera and 51 families. The families with the greatest species richness were Melastomataceae, Fabaceae, Orchidaceae, Asteraceae, Myrtaceae, Euphorbiaceae, and Apocynaceae. Similarity analyses between the arboreal components of the gallery forest of the Mandassaia River and other gallery forests previously examined within the Santo Antonio basin indicated that geographical distance was the most important factor, as closer areas had close similarity values regardless of their altitude. Indicator species for the riparian forests of the Santo Antônio River basin in the Chapada Diamantina include Alchornea triplinervia, Clusia nemorosa, Simarouba amara, Tapirira guianensis, and Vochysia pyramidalis.

Key words: floristic, Chapada Diamantina, gallery forest.

\section{INTRODUÇÃOO}

Estudos florísticos em florestas ciliares têm revelado heterogeneidade na composição e estruturação das espécies em diferentes escalas espaciais. Em escala geográfica, Oliveira-Filho \& Ratter (1995) sugeriram que a rede de florestas de galeria do Brasil Central funciona como corredores ecológicos atuais e pretéritos interligando a floresta amazônica à floresta atlântica, no sentido noroeste-sudeste. A sugestão é exemplificada por Funch (1997), em um estudo com floresta ciliar na Chapada Diamantina, BA, no qual foram registradas espécies consideradas típicas de floresta ciliar e/ou floresta de galeria, por serem muito frequentes nestes ambientes, como Calophyllum brasiliense Cambess., Hirtella glandulosa Spreng., Siparuna guianensis

Artigo recebido em 09/2007. Aceito para publicação em 05/2009.

${ }^{1}$ Universidade Estadual de Feira de Santana, Depto. Ciências Biológicas, Lab. Taxonomia Vegetal, Rod. BR 116, km 03, Feira de Santana, BA, 44031-460, Brasil.

${ }^{2}$ Universidade Federal Rural de Pernambuco, Depto. Biologia, Área de Botânica, R. D. Manoel de Medeiros s/n, Recife, PE, 52171-900, Brasil.

${ }^{3}$ Autor para correspondencia: ligiafunch@yahoo.com.br 
Aubl., Protium heptaphyllum (Aubl.) March., Richeria grandis Vahl e Tapirira guianensis Aubl., as quais distribuem-se da amazônia até a floresta atlântica, atravessando o Brasil Central pelas florestas de galeria e algumas delas alcançando as florestas de brejo, em meio à vegetação de caatinga.

Em escala local, comparações florísticas entre remanescentes de florestas ciliares espacialmente próximas têm mostrado que essas áreas podem ser diversas entre si, com valores de similaridade muito baixos (OliveiraFilho et al. 1990; Felfili \& Silva-Júnior 1992; Felfili et al. 1994; Durigan \& Leitão-Filho 1995; Meguro et al. 1996; Silva-Júnior et al. 1998). Diferentes autores têm demonstrado que a diversidade florística entre as florestas ciliares reflete os fatores ambientais locais como luz e umidade, os quais podem ser influenciados pela altitude, resultando em uma heterogeneidade ambiental gradual ao longo do curso dos rios (Galivanes et al. 1992; Carvalho et al. 1995; Felfili 1995). Especificamente para a bacia hidrográfica Santo Antônio, na Chapada Diamantina, podemos citar os levantamentos em diferentes altitudes realizados por Funch (1997) e Stradman (1997, 2000).

Assim, este estudo visa caracterizar a composição florística de um trecho de floresta ciliar do rio Mandassaia, também pertencente à bacia Santo Antônio, localizada no município de Lençóis, avaliando o grau de semelhança florística com outras florestas ciliares presentes na mesma bacia. Espera-se que áreas próximas e com altitudes distintas tenham floras diferenciadas.

\section{Materiais e Métodos Área de Estudo}

A Chapada Diamantina está inserida na porção baiana da Cadeia do Espinhaço, dividida em várias serras, entre elas, a Serra do Sincorá (CPRM 1994). Na Serra do Sincorá, que ocupa a parte central da borda oriental da chapada (IBGE 1993), situa-se o Parque Nacional da Chapada Diamantina, localizado entre as coordenadas geográficas $12^{\circ} 25^{\prime}-13^{\circ} 20^{\prime} \mathrm{S}$ e $41^{\circ} 35^{\prime}-41^{\circ} 20^{\prime} \mathrm{W}$ (Funch \& Harley 2007). A Chapada Diamantina pode ser considerada como a maior fonte de recursos hídricos da região. Na bacia Santo Antônio, tributária mais importante da bacia do Paraguaçu, nasce o rio Mandassaia localizado no município de Lençóis, dentro dos limites do Parque Nacional da Chapada Diamantina, a ca. $900 \mathrm{~m}$ de altitude (Fig. 1). Seu regime é perene, com volume de água variando de torrencial, na época das chuvas, a muito baixo nos períodos mais secos, tendo o curso total cerca de $15 \mathrm{~km}$ até a foz no rio São José. Os solos da Chapada Diamantina são derivados do metamorfismo regional fraco e insipiente sofrido pelas rochas, sendo predominantemente litólicos distróficos ou álicos, associados a afloramentos de rochas (RADAMBRASIL 1981). O clima característico da região é mesotérmico, do tipo $C w b$ com um máximo de chuvas no verão (novembro, dezembro e janeiro) e um máximo secundário em março-abril (RADAMBRASIL 1981). Nos meses de agosto a novembro há formação de uma estação marcadamente seca. A média de precipitação total anual está acima de $1000 \mathrm{~mm}$ e a temperatura média anual varia de $22^{\circ} \mathrm{C}$ a $25^{\circ} \mathrm{C}$, ficando as mínimas anuais médias em torno de $15^{\circ} \mathrm{C}$ (Funch et al. 2002).

As expedições à campo ocorreram entre janeiro de 2000 e junho de 2001, com coletas mensais. O levantamento florístico foi feito considerando-se as espécies de angiospermas, em fase reprodutiva, no trecho do rio situado a $12^{\circ} 33^{\prime} \mathrm{S}-41^{\circ} 25^{\prime} \mathrm{W}$, a $800 \mathrm{~m}$ de altitude. O material coletado foi depositado no herbário HUEFS. Aidentificação do material foi realizada com auxílio de bibliografia especializada, monografias e floras da região (Harley \& Simmons 1986; Stannard 1995), e por comparação com exsicatas previamente identificadas do HUEFS. Neste trabalho, as famílias foram apresentadas de acordo com o APG II (Souza \& Lorenzi 2008). Para a análise de similaridade florística, aqui baseada apenas no componente arbóreo, utilizou-se o índice de similaridade de Jaccard (Müeller-Dombois \& Ellenberg 1974), a fim de comparar a área de estudo e os levantamentos 


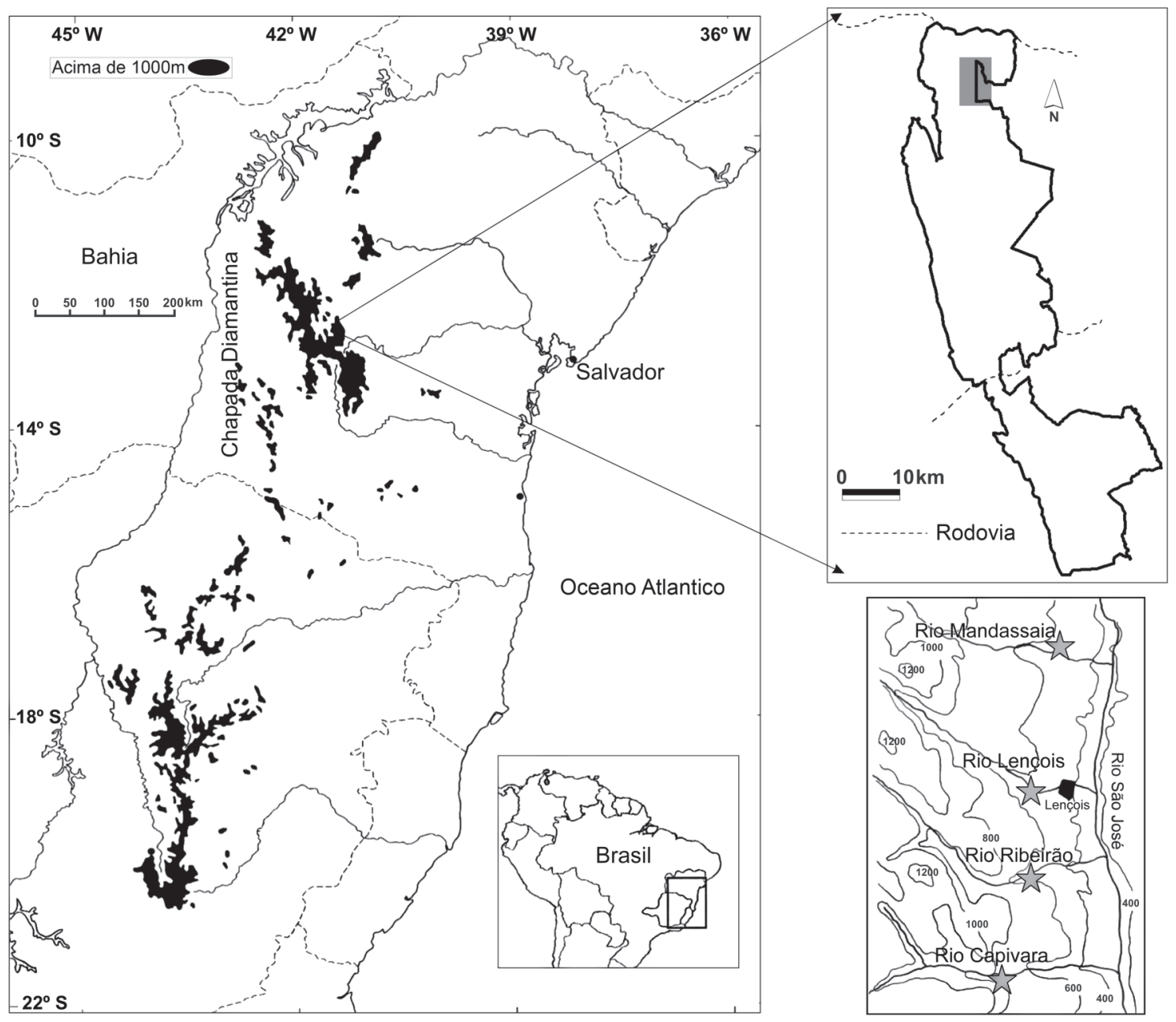

Figura 1 - Localização geográfica da Chapada Diamantina, indicando no perímetro do Parque Nacional da Chapada Diamantina a área de abrangência da bacia Santo Antônio. Em detalhe, a localização dos rios Mandassaia, Lençóis, Ribeirão e Capivara. Figure 1 - Localization of Chapada Diamantina, showing the limits of the National Park of Chapada Diamantina, with Santo Antônio river bay. Detail shows Mandassaia, Lençóis, Ribeirão and Capivara rivers.

em florestas ciliares da borda oriental da Chapada Diamantina, realizados por Funch (1997) no rio Lençóis e por Stradmann $(1997,2000)$ nos rios Ribeirão e Capivara (Fig. 1). Vale destacar que tais florestas ciliares classificam-se como florestas estacionais sub-montanas e montanas, de acordo com Veloso et al. (1991), situadas entre $12^{\circ} 33^{\prime}-12^{\circ} 37^{\prime}$ 'S e 41 $24^{\prime}-41^{\circ} 22^{\prime} \mathrm{W}$, a 450500 m (rios Lençóis e Capivara) e 800 m de altitude (rio Ribeirão), em declividades semelhantes, até $10^{\circ}$, sob regime anual de chuvas de aproximadamente $1200 \mathrm{~mm}$, e se apresentam como faixas estreitas, 5-25 m de largura, que acompanham as margens dos rios, em solos constantemente úmidos (Funch et al. 2008).

\section{Resultados e Discussão}

Foram registradas 116 espécies, distribuídas em 96 gêneros e 51 famílias. Na Tabela 1 estão listadas as espécies coletadas na floresta ciliar do rio Mandassaia, ordenadas por família, com seus respectivos hábitos, número do coletor, estrato de ocorrência e habitat. As famílias com maior riqueza de espécies na floresta ciliar do rio Mandassaia são Melastomataceae, Fabaceae e Orchidaceae (sete espécies cada), seguidas por Asteraceae e Myrtaceae (seis espécies cada) e Apocynaceae (cinco espécies). O somatório da riqueza destas famílias representa $33 \%$ do total das espécies levantadas neste estudo. Em termos de hábito foram encontradas 51 (44\%) 
espécies arbóreas, 24 (21\%) espécies arbustivas, $24(21 \%)$ herbáceas, nove $(8 \%)$ epífitas, quatro (3\%) trepadeiras e quatro (3\%) hemiparasitas. A menor proporção de espécies herbáceas em relação ao total de espécies levantadas representa um indicativo de bom estado de conservação desta formação ciliar (Felfili 2000).

Em trechos do rio onde há afloramento de rocha e atividades garimpeiras, ocorre uma vegetação aberta, com porte herbáceoarbustivo, de transição entre a floresta e o campo rupestre adjacente, alcançando até quatro metros de altura. Tais áreas apresentam espécies que não são comuns à vegetação ciliar registrada em outros estudos (Funch 1997; Stradmann 1997, 2000), como Syagrus harleyi e Paralychnophora bicolor.

A floresta ciliar se apresenta constituída de um estrato superior com ca. 15 metros, compondo o dossel e com algumas espécies arbóreas emergentes; um sub-dossel com árvores que variam entre 6 a 8 metros; e um sub-bosque com poucos indivíduos arbustivos e algumas ervas, além de indivíduos jovens das espécies que ocupam os estratos superiores.

As espécies mais abundantes que compõem o estrato superior da fisionomia florestal adjacente ao curso do rio são: Alchornea triplinervia e Maprounea guianensis (Euphorbiaceae); Clusia nemorosa (Clusiaceae); Calyptranthes pulchella e Myrcia detergens (Myrtaceae); Tapirira guianensis (Anacardiaceae); e Balizia pedicellaris (Fabaceae). Como principal emergente destaca-se Aspidosperma discolor (Apocynaceae). A maioria destas espécies compõe o estrato superior das florestas ciliares de outros rios que formam a bacia Santo Antônio. Além disso, este conjunto de espécies é citado para a maioria dos levantamentos florísticos realizados no Brasil extra-amazônico (Rodrigues \& Nave 2000).

O sub-dossel é descontínuo, destacando-se a família Myrtaceae, com as espécies Eugenia subterminalis, Myrcia blanchetiana, $M$. vestita e a espécie subespontânea Syzygium jambos. As outras famílias que foram bem representadas no sub-dossel foram: Melastomataceae, com Miconia chartaceae, Tibouchina macrochiton e $T$. pereirae; e Clusiaceae com Clusia melchiorii e $C$. obdeltifolia.

O estrato herbáceo-arbustivo é composto por poucos indivíduos arbustivos de 2 a $4 \mathrm{~m}$, ervas, e indivíduos jovens das espécies que ocupam os estratos superiores. A família Asteraceae é uma das mais ricas em espécies arbustivas, com 7 espécies encontradas, entre elas Paralychnophora bicolor e Moquinia racemosa. Paralychnophora bicolor foi encontrada nos três rios estudados na bacia Santo Antônio (Funch 1997; Stradmann 1997, 2000), e em trechos com maior influência antrópica do rio Mandassaia. Moquinia racemosa é citada pela primeira vez para florestas ciliares na bacia Santo Antônio, tendo sido registrada anteriormente em florestas ciliares da Serra do Ambrósio (Meguro et al. 1994; Pirani et al. 1994) e da Serra do Cipó (Meguro et al. 1996), na Cadeia do Espinhaço. Fabaceae participa também com destaque na composição, com espécies de hábito arbustivo como Calliandra hirtiflora, Calliandra parvifolia e Chamaecrista zygophylloides.

Sobre os afloramentos de rocha e em antigas áreas de atividades garimpeiras adjacentes ao rio Mandassaia, são comuns espécies de Melastomataceae, como Marcetia bahiensis, Miconia theaezans e Trembleya parviflora, frequentes nos campos rupestres da Chapada Diamantina(Harley 1995). Acomposição florística desta fisionomia é ainda formada por representantes de Orchidaceae, Eriocaulaceae, Bromeliaceae, Velloziaceae, Cyperaceae, Xyridaceae, Cactaceae (ver Tab. 1), cujas espécies estão presentes na maioria das formações ciliares e também na vegetação de campo rupestre da região (Harley \& Simmons 1986; Stannard 1995; Funch 1997; Stradmann 1997, 2000; Conceição \& Giulietti 2002).

Espécies de Orchidaceae, Bromeliaceae e Araceae estão presentes principalmente sobre as pedras que formam as margens do rio, nas porções mais abertas da vegetação. 
Tabela 1-Relação das famílias e espécies coletadas por Ribeiro-Filho na vegetação ciliar do rio Mandassaia, Lençóis, Chapada Diamantina, Bahia. Número do coletor (NC), hábito (Ho), árvore (Ar), arbusto (Ab), erva (Er), trepadeira (Tp), epífita (Ep), hemiparasita (He), estratos (ES), emergente (EM), dossel (DO), sub-dossel (SD) e herbáceo-arbustivo (HA), habitat (Ht), floresta ciliar (FC) e campo rupestre (CR).

Table 1 - Plant families and species collected by Ribeiro-Filho in the riparian forest at Mandassaia river, municipality of Lençóis, Chapada Diamantina, Bahia. Number of the collector (NC), habit (Ho), tree (Ar), shrub (Ab), herb (Er), climbers (Tp), epiphyte (Ep), hemiparasite (He), strata (ES), emergent (EM), canopy (DO), sub-canopy (SD) and herbaceous and arbustive (HA), habitat (Ht), river forest (FC) e rupestrian fields (CR).

\begin{tabular}{|c|c|c|c|c|}
\hline Família/Espécie & $\mathrm{NC}$ & Ho & ES & $\mathbf{H t}$ \\
\hline \multicolumn{5}{|l|}{ Anacardiaceae } \\
\hline Tapirira guianensis Aubl. & 253 & $\mathrm{Ar}$ & DO & $\mathrm{FC}$ \\
\hline Tapirira obtusa (Benth.) J. D. Mitch. & 29 & $\mathrm{Ar}$ & DO & $\mathrm{FC}$ \\
\hline \multicolumn{5}{|l|}{ Apocynaceae } \\
\hline Aspidosperma discolor A. DC. & 326 & $\mathrm{Ar}$ & EM & $\mathrm{FC}$ \\
\hline Couma rigida Müll. Arg. & 08 & $\mathrm{Ar}$ & SD & $\mathrm{FC}$ \\
\hline Himatanthus lancifolius (Müll. Arg.) Woodson & & $\mathrm{Ar}$ & SD & $\mathrm{FC}$ \\
\hline Mandevilla bahiensis (Woodson) Sales & 248 & $\mathrm{Er}$ & HA & CR \\
\hline Mandevilla coccinea (Hook \& Arn.) Woodson & 265 & $\mathrm{Tp}$ & HA & CR \\
\hline \multicolumn{5}{|l|}{ Aquifoliaceae } \\
\hline Ilex amara Loes & 19 & $\mathrm{Ar}$ & SD & $\mathrm{FC}$ \\
\hline \multicolumn{5}{|l|}{ Araceae } \\
\hline Anthurium scandens (Aubl.) Engl. & 68 & Er & HA & CR \\
\hline Philodendron imbe Schott & 275 & $\mathrm{Er}$ & HA & $\mathrm{CR} / \mathrm{FC}$ \\
\hline \multicolumn{5}{|l|}{ Arecaceae } \\
\hline Syagrus harleyi Glassmann & 267 & $\mathrm{Ab}$ & HA & CR \\
\hline \multicolumn{5}{|l|}{ Asteraceae } \\
\hline Acritopappus hagei R. M. King \& H. Rob. & 22 & $\mathrm{Ab}$ & HA & $\mathrm{CR} / \mathrm{FC}$ \\
\hline Ayapanopsis oblongifolia (Gardner) R. M. King \& H. Rob. & 97 & $\mathrm{Tp}$ & HA & $\mathrm{CR} / \mathrm{FC}$ \\
\hline Baccharis salzmannii DC. & 94 & $\mathrm{Ab}$ & SD & $\mathrm{CR} / \mathrm{FC}$ \\
\hline Moquinia racemosa (Spreng.) DC. & 91 & $\mathrm{Ab}$ & HA & $\mathrm{CR} / \mathrm{FC}$ \\
\hline Paralychnophora bicolor (DC.) MacLeish & 257 & $\mathrm{Ab}$ & HA & $\mathrm{CR} / \mathrm{FC}$ \\
\hline Vernonia cotoneaster (Willd. ex Spreng.) Less. & 48 & $\mathrm{Ab}$ & HA & $\mathrm{CR} / \mathrm{FC}$ \\
\hline \multicolumn{5}{|l|}{ Begoniaceae } \\
\hline Begonia grisea A. DC. & 78 & Er & HA & CR \\
\hline \multicolumn{5}{|l|}{ Bonnetiaceae } \\
\hline Bonnetia stricta (Nees) Nees \& Mart. & 02 & $\mathrm{Ar}$ & SD & $\mathrm{FC}$ \\
\hline \multicolumn{5}{|l|}{ Bignoniaceae } \\
\hline Jacaranda irwinii A. H. Gentry & 300 & $\mathrm{Ar}$ & HA & $\mathrm{CR} / \mathrm{FC}$ \\
\hline \multicolumn{5}{|l|}{ Bromeliaceae } \\
\hline Orthophytum navioides (L. B. Sm.) L. B. Sm. & 269 & Er & HA & CR \\
\hline Tillandsia usneoides (L.) L. & 273 & Epífita & HA & $\mathrm{CR} / \mathrm{FC}$ \\
\hline Tillandsia $\mathrm{sp}$. & 274 & Ep & HA & $\mathrm{CR} / \mathrm{FC}$ \\
\hline Vriesea friburgensis $\mathrm{Mez}$ & 268 & Er & HA & $\mathrm{CR} / \mathrm{FC}$ \\
\hline \multicolumn{4}{|l|}{ Cactaceae } & $\mathrm{FC}$ \\
\hline \multicolumn{5}{|l|}{ Celastraceae } \\
\hline Maytenus mисugensis Car.-Okano & 283 & $\mathrm{Ar}$ & SD & $\mathrm{FC}$ \\
\hline
\end{tabular}


Família/Espécie

Chrysobalanaceae

Licania kunthiana Hook. f.

\section{Clusiaceae}

Calophyllum brasiliense Cambess.

Clusia melchiorii Gleason

Clusia nemorosa G. Mey

Clusia obdeltifolia Bittrich

\section{Convolvulaceae}

Jacquemontia montana (Moric.) Meisn.

Cyperaceae

Lagenocarpus rigidus (Kunth) Nees

Elaeocarpaceae

Sloanea guianensis (Aubl.) Benth.

Ericaceae

Agarista oleifolia var. glabra (Meisn.) Judd

Eriocaulaceae

Paepalanthus bifidus (Schrad.) Kunth

Paepalanthus erigeron Mart.

Paepalanthus spathulatus Körn.

Paepalanthus tortilis (Bong.) Koern.

\section{Fabaceae}

Aeschynomene brevipes Benth.

Balizia pedicellaris (DC.) Barneby \& J.W. Grimes

Calliandra hirtiflora Benth.

Calliandra parvifolia (Hook. \& Arn.) Speg.

Camptosema coriaceum (Nees \& Mart.) Benth.

Centrosema sp.

Chamaecrista zygophylloides var. colligans H.S. Irwin \& Barneby

\section{Euphorbiaceae}

Alchornea triplinervia (Spreng.) Müll. Arg.

Maprounea guianensis Aubl.

Stillingia saxatilis Müll. Arg.

\section{Gentianaceae}

Chelonanthus uliginosus (Griseb.) Gilg

Gesneriaceae

Paliavana tenuiflora Mansf.

Sinningia sp.

\section{Humiriaceae}

Humiria balsamifera Aubl.

Vantanea obovata (Nees \& Mart.) Benth.

\section{Lamiaceae}

Eriope exaltata Harley

Vitex sp.

NC Ho ES Ht

$331 \quad$ Ar $\quad$ DO $\quad$ FC

333 Ar DO FC

299 Ar $\quad$ SD $\quad$ FC

26 Ar $\quad$ DO $\quad$ FC

60 Ar SD CR/FC

102 Tp HA CR

66 Er HA CR

03 Ar $\quad$ DO $\quad$ FC

09 Ar DO CR/FC

71 Er HA CR/FC

107 Er HA CR/FC

108 Er HA CR/FC

104 Er HA CR/FC

266 Er HA CR

43 Ar DO FC

31 Ab HA FC

$40 \quad \mathrm{Ab} \quad \mathrm{HA} \quad \mathrm{CR} / \mathrm{FC}$

$39 \quad \mathrm{Ab} \quad \mathrm{HA} \quad \mathrm{CR}$

55 Tp $\mathrm{HA} \quad \mathrm{CR}$

$70 \mathrm{Ab} \quad \mathrm{HA} \quad \mathrm{CR} / \mathrm{FC}$

$14 \quad \mathrm{Ar} \quad \mathrm{DO} \quad \mathrm{FC}$

203 Ar DO FC

293 Ab SD CR

34 Er HA CR

$35 \mathrm{Ab} \quad \mathrm{HA} \quad \mathrm{CR}$

298 Ab HA CR

17 Ar $\quad$ SD $\quad$ FC

44 Ar HA CR/FC

25 Ar $\quad$ SD $\quad F C$

$\mathrm{Ab} \quad \mathrm{HA} \quad \mathrm{FC}$


Família/Espécie

Lauraceae

Cinnamomum sp.

Ocotea puberula (Rich.) Nees

Lentibulariaceae

Utricularia sp.

Loranthaceae

Psittacanthus robustus (Mart.) Mart.

Struthanthus flexicaulis (Mart. ex Schult. f.) Mart.

Lythraceae

Diplusodon ulei subsp. cilliatus T.B. Cavalc.

Malpighiaceae

Byrsonima sericea DC.

Byrsonima stannardii W. R. Anderson

Verrucularia glaucophylla A. Juss.

Malvaceae

Waltheria cinerescens A. St.-Hil.

Marantaceae

Monotagma plurispicatum (Koern.) K. Schum.

Marcgraviaceae

Norantea guianensis Aubl.

Melastomataceae

Marcetia bahiensis (Brade \& Markgr.) Wurdack

Miconia chartacea Triana

Miconia theaezans (Bonpl.) Cogn.

Microlicia sp.

Tibouchina macrochiton Cogn.

Tibouchina pereirae Brade \& Markgr.

Trembleya parviflora (D. Don) Cogn.

Myrsinaceae

Myrsine guianensis (Aubl.) Kuntze

Myrsine umbellata G. Don

Myrtaceae

Calyptranthes pulchella DC.

Eugenia subterminalis DC.

Myrcia blanchetiana (O. Berg.) Mattos

Myrcia detergens Miq.

Myrcia vestita DC.

Syzygium jambos (L.) Alston

Ochnaceae

Sauvagesia sp.

Olacaceae

Heisteria perianthomega (Vell.) Sleumer

Orchidaceae

Bulbophyllum ipanemense Hoehne

Cattleya elongata Barb. Rodr.

NC Ho ES Hit

Ar $\quad$ SD $\quad \mathrm{FC}$

316 Ar $\quad$ SD $\quad$ FC

42 Er HA CR

$11 \mathrm{He}$ HA CR/FC

$12 \mathrm{He} \mathrm{HA} \mathrm{CR} / \mathrm{FC}$

$287 \mathrm{Ab} \quad \mathrm{HA} \quad \mathrm{CR} / \mathrm{FC}$

23 Ar DO FC

318 Ab HA CR

21 Ab HA CR

311 Ar $\mathrm{HA} \quad \mathrm{FC}$

272 Er $\quad$ HA $\quad$ FC

$15 \quad \mathrm{Ar} \quad \mathrm{SD} \quad \mathrm{FC}$

$47 \quad \mathrm{Ab} \quad \mathrm{HA} \quad \mathrm{CR}$

304 Ar SD $\mathrm{FC}$

16 Ar SD FC

75 Ab $\mathrm{HA} \quad \mathrm{CR}$

07 Ar SD CR

270 Ar SD FC

$47 \quad \mathrm{Ab} \quad \mathrm{HA} \quad \mathrm{CR}$

$280 \quad \mathrm{Ab} \quad \mathrm{HA} \quad \mathrm{FC}$

295 Ar DO FC

328 Ar $\quad$ DO $\quad$ FC

$327 \quad \mathrm{Ab} \quad \mathrm{SD} \quad \mathrm{FC}$

30 Ar $\quad$ SD $\quad$ FC

329 Ar DO FC

$276 \quad \mathrm{Ab} \quad \mathrm{SD} \quad \mathrm{FC}$

259 Ar $\quad$ SD $\quad$ FC

69 Er HA CR

13 Ar DO FC

101 Er $\quad$ HA CR

59 Er HA CR




\begin{tabular}{|c|c|c|c|c|}
\hline Família/Espécie & $\mathrm{NC}$ & Ho & ES & $\mathbf{H t}$ \\
\hline \multicolumn{5}{|l|}{ Orchidaceae } \\
\hline Epistephium lucidum Cogn. & 51 & Ep & HA & $\mathrm{CR}$ \\
\hline Pleurothallis hamosa Barb. Rodr. & 332 & $\mathrm{Er}$ & HA & $\mathrm{CR}$ \\
\hline Pleurothallis ochreata Lindl. & 62 & Er & HA & $\mathrm{CR}$ \\
\hline Sobralia liliastrum Lindl. & 58 & $\mathrm{Er}$ & HA & $\mathrm{CR}$ \\
\hline Sobralia sp. & 258 & Er & HA & $\mathrm{FC}$ \\
\hline \multicolumn{5}{|l|}{ Orobanchaceae } \\
\hline Esterhazya splendida J. C. Mikan & 76 & $\mathrm{Ab}$ & HA & $\mathrm{CR}$ \\
\hline \multicolumn{5}{|l|}{ Phyllanthaceae } \\
\hline Phyllanthus klotzschianus Müll. Arg. & 46 & Er & HA & $\mathrm{CR}$ \\
\hline Richeria grandis Vahl & 204 & $\mathrm{Ar}$ & DO & $\mathrm{FC}$ \\
\hline \multicolumn{5}{|l|}{ Piperaceae } \\
\hline Piper sp. & 277 & Er & HA & $\mathrm{CR} / \mathrm{FC}$ \\
\hline \multicolumn{5}{|l|}{ Rubiaceae } \\
\hline Alibertia concolor (Cham.) K. Schum. & 334 & $\mathrm{Ar}$ & $\mathrm{SD}$ & $\mathrm{FC}$ \\
\hline Declieuxia aspalathoides Müll. Arg. & 255 & $\mathrm{Ab}$ & HA & $\mathrm{CR}$ \\
\hline Diodia sarmentosa $\mathrm{Sw}$. & 103 & Er & HA & $\mathrm{CR}$ \\
\hline Posoqueria latifolia (Rudge) Roem. \& Schult. & 18 & $\mathrm{Ar}$ & DO & $\mathrm{FC}$ \\
\hline \multicolumn{5}{|l|}{ Rutaceae } \\
\hline Hortia brasiliana Vand. ex DC. & 279 & $\mathrm{Ar}$ & DO & $\mathrm{FC}$ \\
\hline \multicolumn{5}{|l|}{ Salicaceae } \\
\hline Casearia sp. & 20 & $\mathrm{Ar}$ & $\mathrm{SD}$ & $\mathrm{FC}$ \\
\hline \multicolumn{5}{|l|}{ Santalaceae } \\
\hline Phoradendron sp. 1 & 28 & $\mathrm{He}$ & HA & $\mathrm{CR} / \mathrm{FC}$ \\
\hline Phoradendron sp. 2 & 61 & $\mathrm{He}$ & HA & $\mathrm{CR} / \mathrm{FC}$ \\
\hline \multicolumn{5}{|l|}{ Sapotaceae } \\
\hline Pouteria ramiflora (Mart.) Radlk. & 262 & $\mathrm{Ar}$ & DO & $\mathrm{FC}$ \\
\hline $\begin{array}{l}\text { Sideroxylon obtusifolium (Humb. ex Roem. } \\
\text { \& Schult.) T. D. Penn. }\end{array}$ & 32 & $\mathrm{Ab}$ & HA & $\mathrm{CR} / \mathrm{FC}$ \\
\hline \multicolumn{5}{|l|}{ Simaroubaceae } \\
\hline Simarouba amara Aubl. & 330 & $\mathrm{Ar}$ & DO & $\mathrm{FC}$ \\
\hline \multicolumn{5}{|l|}{ Theaceae } \\
\hline Gordonia fruticosa (Schrad.) H. Keng & 92 & $\mathrm{Ar}$ & DO & $\mathrm{FC}$ \\
\hline Ternstroemia alnifolia Wawra & 36 & $\mathrm{Ar}$ & DO & $\mathrm{FC}$ \\
\hline Ternstroemia candolleana Wawra & 325 & $\mathrm{Ar}$ & $\mathrm{DO}$ & $\mathrm{FC}$ \\
\hline \multicolumn{5}{|l|}{ Velloziaceae } \\
\hline Vellozia canelinha Mello-Silva & 291 & Er & HA & $\mathrm{CR}$ \\
\hline \multicolumn{5}{|l|}{ Verbenaceae } \\
\hline Stachytarpheta crassifolia Schrad. & 53 & $\mathrm{Ab}$ & HA & $\mathrm{CR}$ \\
\hline \multicolumn{5}{|l|}{ Vochysiaceae } \\
\hline Qualea sp. & & $\mathrm{Ar}$ & SD & $\mathrm{FC}$ \\
\hline Vochysia pyramidalis Mart. & 57 & $\mathrm{Ar}$ & DO & $\mathrm{FC}$ \\
\hline Vochysia thyrsoidea Pohl & 246 & $\mathrm{Ab}$ & HA & $\mathrm{FC}$ \\
\hline \multicolumn{5}{|l|}{ Xyridaceae } \\
\hline Xyris sp. & 27 & $\mathrm{Er}$ & HA & $\mathrm{CR}$ \\
\hline
\end{tabular}


Porém, ocorrem também sobre as árvores na floresta ciliar, como Sobralia sp. (Orchidaceae) e Vriesea friburgensis (Bromeliaceae).

Entre as espécies epifíticas destacam-se as do gênero Tillandsia (Bromeliaceae), por sua abundância na área. As hemiparasitas, embora estejam representadas por quatro espécies na vegetação ciliar do rio Mandassaia, não são comuns nas demais áreas junto aos outros rios estudados na região. As famílias com maior riqueza específica na floresta ciliar do rio Mandassaia foram as mesmas dos demais rios estudados anteriormente (Funch 1997; Stradmann 1997, 2000), porém com número de espécies diferenciados. Todavia, tratam-se de famílias que aparecem em destaque na maioria dos levantamentos florísticos nas diversas formações vegetacionais brasileiras (Absy et al. 1986; Peixoto \& Gentry 1990; Carvalho et al. 1995).

Os resultados encontrados na análise de similaridade florística entre a floresta ciliar do rio Mandassaia e outras florestas ciliares da bacia Santo Antônio estudadas anteriormente (Funch 1997; Stradmann 1997, 2000) evidenciaram acentuada heterogeneidade florística entre as florestas, uma vez que o índice de similaridade de Jaccard entre o rio Mandassaia e as demais áreas foi $22 \%$; com os rios Lençóis e Ribeirão, $22 \%$; e $15 \%$ com o rio Capivara.

Tabela 2 - Lista das espécies arbóreas presentes nos levantamentos florísticos das florestas ciliares da bacia Santo Antônio: rio Mandassaia (este estudo), rio Lençóis (Funch 1997) e rios Ribeirão e Capivara (Stradmann 1997, 2000).

Table 2 - List of tree species founded in the floristic surveyes of the riparian Forest from the Santo Antônio river bay: Mandassaia river (this study), Lençóis river (Funch 1997) and Ribeirão and Capivara rivers (Stradmann 1997, 2000).

\begin{tabular}{|c|c|c|c|c|}
\hline Espécie & Mandassaia & Lençóis & Ribeirão & Capivara \\
\hline Alchornea triplinervia (Spreng.) Müll. Arg. & $\mathrm{X}$ & $\mathrm{x}$ & $\mathrm{x}$ & $\mathrm{x}$ \\
\hline Balizia pedicellaris (DC.) Barneby \& Grimes & $\mathrm{x}$ & $\mathrm{x}$ & $\mathrm{x}$ & $\mathrm{x}$ \\
\hline Bonnetia stricta (Nees) Nees \& Mart. & $\mathrm{x}$ & $\mathrm{x}$ & $\mathrm{x}$ & $\mathrm{x}$ \\
\hline Clusia nemorosa G. Mey & $\mathrm{x}$ & $\mathrm{x}$ & $\mathrm{x}$ & $\mathrm{x}$ \\
\hline Eugenia subterminalis DC. & $\mathrm{x}$ & $\mathrm{x}$ & $\mathrm{x}$ & $\mathrm{x}$ \\
\hline Heisteria perianthomega (Vell.) Sleumer & $\mathrm{x}$ & $\mathrm{x}$ & $\mathrm{x}$ & $\mathrm{x}$ \\
\hline Licania kunthiana Hook. f. & $\mathrm{x}$ & $\mathrm{x}$ & $\mathrm{x}$ & $\mathrm{x}$ \\
\hline Myrcia blanchetiana (O. Berg.) Mattos & $\mathrm{x}$ & $\mathrm{x}$ & $\mathrm{x}$ & $\mathrm{x}$ \\
\hline Pouteria ramiflora (Mart.) Radlk. & $\mathrm{x}$ & $\mathrm{x}$ & $\mathrm{x}$ & $\mathrm{x}$ \\
\hline Simarouba amara Aubl. & $\mathrm{x}$ & $\mathrm{x}$ & $\mathrm{x}$ & $\mathrm{x}$ \\
\hline Tapirira guianensis Aubl. & $\mathrm{x}$ & $\mathrm{x}$ & $\mathrm{x}$ & $\mathrm{x}$ \\
\hline Vochysia pyramidalis Mart. & $\mathrm{x}$ & $\mathrm{x}$ & $\mathrm{x}$ & $\mathrm{x}$ \\
\hline Byrsonima sericea DC. & $\mathrm{x}$ & $\mathrm{x}$ & & $\mathrm{x}$ \\
\hline Richeria grandis Vahl & $\mathrm{x}$ & $\mathrm{x}$ & & $\mathrm{x}$ \\
\hline Calophyllum brasiliense Cambess. & $\mathrm{X}$ & $\mathrm{x}$ & $\mathrm{x}$ & \\
\hline Calyptranthes pulchella DC. & $\mathrm{X}$ & $\mathrm{X}$ & $\mathrm{X}$ & \\
\hline Couma rigida Müll. Arg. & $\mathrm{x}$ & $\mathrm{x}$ & $\mathrm{x}$ & \\
\hline Humiria balsamifera Aubl. & $\mathrm{x}$ & $\mathrm{x}$ & $\mathrm{x}$ & \\
\hline Miconia chartacea Triana & $\mathrm{x}$ & $\mathrm{x}$ & $\mathrm{x}$ & \\
\hline Myrsine umbellata Mart. & $\mathrm{x}$ & $\mathrm{x}$ & $\mathrm{x}$ & \\
\hline Sloanea guianensis (Aubl.) Benth. & $\mathrm{x}$ & $\mathrm{x}$ & $\mathrm{x}$ & \\
\hline Tapirira obtusa (Benth.) J.D. Mitch. & $\mathrm{x}$ & $\mathrm{x}$ & $\mathrm{x}$ & \\
\hline
\end{tabular}


Muitos estudos em florestas ciliares sugerem que mesmo florestas próximas podem apresentar composição florística distinta, dependendo das condições ambientais predominantes em cada uma delas (Felfili et al. 1994; Oliveira-Filho \& Ratter 1995; Walter 1995; Rezende et al. 1997). Segundo Rodrigues (1992), a altitude desempenha um importante papel na composição florística e na estrutura das comunidades florestais.

Se considerarmos distância geográfica e altitude como fatores que podem influenciar na similaridade entre áreas, pode-se concluir que no caso da bacia Santo Antônio a distância geográfica foi o fator mais importante, uma vez que as áreas mais próximas evidenciaram valores de similaridade semelhantes, independente de sua altitude, enquanto o do rio Capivara, que representa o sítio mais distante da área de estudo, foi ainda mais baixo.

Nos demais levantamentos florísticos realizados na bacia Santo Antônio ocorreram diversas espécies comuns à área de estudo, embora um número considerável (59) tenha sido exclusivo da área de estudo. Os quatro rios apresentaram 10 espécies arbóreas em comum, e 13 espécies arbóreas ocorreram em pelo menos três dos quatro rios estudados na bacia (Tab. 2). Algumas destas estão presentes em grande parte dos levantamentos florísticos realizados em florestas ciliares extra-amazônicas, como: Tapirira guianensis, Alchornea triplinervia e Calophyllum brasiliensis, consideradas espécies típicas de floresta ciliar (Rodrigues \& Nave 2000). Dentre essas, Funch (1997) indicou as espécies Licania kunthiana, Couma rigida, Richeria grandis, Heisteria perianthomega, Vochysia pyramidalis e Humiria balsamifera, como restritas às florestas ciliares na região. É notável que embora estas espécies sejam de ampla distribuição geográfica, estas se encontram associadas aos cursos d'água nas quatro áreas estudadas da bacia Santo Antônio, sendo por tanto potenciais indicadoras de florestas ciliares para a região oriental da Chapada Diamantina.
Conclui-se que a distância geográfica, independente de sua altitude, foi o fator mais importante para explicar as semelhanças florísticas ao longo da bacia do Santo Antônio. Tal fato reforça a importância de estudos locais para estabelecer modelos de manejo, recuperação e conservação de florestas ciliares nesta bacia hidrográfica (Rezende 1998).

\section{REFERÊNCIAS BIBLIOGRÁFICAS}

Absy, M. L.; Prance, G. T. \& Barbosa, E. M. 1986. Inventário florístico de floresta natural na área da estrada Cuiabá - Porto Velho (BR 364). Acta Amazonica 16/17 (Suppl.): 85-122.

Carvalho, D. A.; Oliveira-Filho, A. T.; Vilela, E. A. \& Gavilanes, M. L. 1995. Estrutura fitossociológica de mata ripária do alto rio Grande (Bom Sucesso/MG). Revista Brasileira de Botânica 18(1): 39-50.

CPRM. 1994. Projeto Chapada Diamantina; Parque Nacional da Chapada Diamantina - BA: informações básicas para a gestão territorial - Diagnóstico do meio físico e da vegetação. CPRM, Salvador, 116p.

Conceição, A. A. \& Giulietti, A. M. 2002. Composição florística e aspectos estruturais de campo rupestre em dois platôs do Morro do Pai Inácio, Chapada Diamantina, Bahia, Brasil. Hoehnea 29(1): 37-48.

Durigan, G. \& Leitão-Filho, H. F. 1995. Florística e fitossociologia de matas ciliares do oeste paulista. Revista do Instituto Florestal 7(2): 197-239.

Felfili, J. M. 1995. Diversity, structure and dynamics of gallery forest in Central Brazil. Vegetatio 117: 1-15.

Felfili, J. M. 2000. Crescimento, recrutamento e mortalidade nas matas de galeria do planalto central. In: Cavalcanti, T. B. \& Walter, B. M. T. (org.). Tópicos atuais em Botânica: palestras convidadas do $51^{\circ}$ Congresso Nacional de Botânica. Embrapa Recursos Genéticos e Biotecnologia, Brasília. Pp. 152-158.

Felfili, J. M. \& Silva-Júnior, M. C. 1992. Floristic composition, phytosociology and comparison of cerrado and gallery forest 
at Fazenda Água Limpa, Federal District, Brazil. In: Furley, A. P.; Proctor, J. \& Ratter, J. A. (eds.). Nature and dynamics of forest and savanna boundaries. Chapman \& Hall, London. Pp. 393-415.

Felfili, J. M.; Silva-Júnior, M. C.; Rezende, A. V.; Machado, J. M. B.; Walter, B. M. T.; Silva, P. E. \& Hay, J. D. 1994. Vegetação arbórea. In: Felfili, J. M.; Filgueiras, T. S.; Haridasan, M.; Silva-Júnior, M. C.; Mendonça, R. C. \& Rezende, A. V. (eds.). Projeto biogeografia do bioma cerrado: vegetação e solos. Cadernos de Geociências do IBGE, Rio de Janeiro. Pp. 75-166.

Funch, L. S. 1997. Composição florística e fenologia de mata ciliar e mata de encosta, adjacentes ao rio Lençóis, Lençóis, BA. Tese de Doutorado. Universidade Estadual de Campinas, Campinas, 298p.

Funch, L. S.; Funch, R. R. \& Barroso, G. M. 2002. Phenology of gallery and montane forest in the Chapada Diamantina, Bahia, Brazil. Biotropica 34: 40-50.

Funch, L. S.; Rodal, M. J. N. \& Funch, R. R. 2008. Floristic aspects of forests of the Chapada Diamantina, Bahia, Brazil. In: Thomas, W. \& Britton, E. G. (eds.). The Atlantic coastal forest of northeastern Brazil. New York Botanical Garden Press, New York. Pp. 193-220.

Funch, R. R. \& Harley, R. M. 2007. Reconfiguring the boundaries of the Chapada Diamantina National Park (Brazil) using ecological criteria in the context of a human-dominated landscape. Landscape and Urban Planning 83: 355-362.

Galivanes, M. L.; Brandão, M.; Oliveira-Filho, A. T.; Almeida, R. J.; Mello, J. M. \& Avezum, F. F. 1992. Flórula da Reserva Biológica do Poço Bonito, Lavras, MG. II - Formação florestal. Daphne 2: 14-26.

Harley, R. M. 1995. Introduction. In: Stannard, B. L. (ed.). Flora of the Pico das Almas, Chapada Diamantina, Bahia, Brazil. Royal Botanic Gardens, Kew. Pp. 1-40.

Harley, R. M. \& Simmons, N. A. 1986. Flórula de Mucugê, Chapada Diamantina, Bahia, Brazil. Royal Botanic Gardens, Kew, 123p.
IBGE. 1993. Diagnóstico geoambiental e sócioeconômico da bacia do rio Paraguaçu BA. Diretrizes gerais para a ordenação territorial. Série Estudos e Pesquisas em Geociências 1, Rio de Janeiro, 124p.

Meguro, M.; Pirani, J. R.; Giulietti, A. M. \& Mello-Silva, R. 1994. Phytophysiognomy and composition of the vegetation of Serra do Ambrósio, Minas Gerais, Brazil. Revista Brasileira de Botânica 17(2): 149-166.

Meguro, M.; Pirani, J. R.; Mello-Silva, R. \& Giulietti, A. M. 1996. Caracterização florística e estrutural de matas ripárias e capões de altitude da Serra do Cipó, Minas Gerais. Boletim de Botânica da Universidade de São Paulo 15: 13-29.

Müeller-Dombois, D. \& Elenberg, H. 1974. Aims and methods of vegetation ecology. John Wiley \& Sons, New York, 547p.

Oliveira-Filho, A. T. \& Ratter, J. A. 1995. A study of the origin of central Brazilian forests by the analysis of plant species distribution patterns. Edinburgh Journal of Botany 52: 103-117.

Oliveira-Filho, A. T.; Ratter, J. A. \& Shepherd, G. J. 1990. Floristic composition and community structure of a central Brazilian gallery forest. Flora 184: 103-117.

Peixoto, A. L. \& Gentry, A. 1990. Diversidade e composição florística da mata de tabuleiro na Reserva Florestal de Linhares (ES, Brasil). Revista Brasileira Botânica 13: 19-25.

Pirani, J. R.; Giulietti, A. M.; Mello-Silva, R. \& Meguro, M. 1994. Checklist and patterns of geographic distribution of the vegetation of Serra do Ambrósio, Minas Gerais, Brazil. Revista Brasileira Botânica 17(2): 133-147.

RADAMBRASIL. 1981. Levantamento de recursos naturais. Folha SD.24 - Salvador; Geologia, geomorfologia, pedologia, vegetação e uso potencial da terra. Ministério das Minas e Energia, Rio de Janeiro, 624p.

Rezende, A. V. 1998. Importância das matas de galeria: manutenção e recuperação. In: Ribeiro, J. F. (ed.). Cerrado: matas de galeria. EMBRAPA/CPAC, Planaltina. Pp. 78-95. 
Rezende, A. V.; Felfili, J. M.; Silva, P. E. N.; Silva-Júnior, M. C. \& Silva, M. A. 1997. Comparison of gallery forests on welldrained soils on the Veadeiros Plateaux, Goiás, Brazil. In: Imaña-Encinas, J. \& Klein, C. (eds.). Proceedings of international symposium on assessment and monitoring of forests in tropical dry regions with special reference to gallery forests. Universidade de Brasília, Brasília. Pp. 365-378.

Rodrigues, R. R. 1992. Análise da vegetação às margens do Rio Passa Cinco, Ipeúna, SP. Tese de Doutorado. Universidade Estadual de Campinas, Campinas, 334p.

Rodrigues, R. R. \& Nave, A. G. 2000. Heterogeneidade florística da matas ciliares. In: Rodrigues, R. R. \& Leitão Filho, H.F. (eds.) Matas ciliares: conservação e recuperação. EdUSP/Fapesp, São Paulo. Pp. 45-71.

Silva-Júnior, M. C.; Felfili, J. M.; Nogueira, P. E. \& Rezende, A. V. 1998. Análise florística das matas de galeria no Distrito Federal. In: Ribeiro, J. F. (ed.). Cerrado: matas de galeria. EMBRAPA/CPAC, Planaltina. Pp. 32-58.

Souza, V. C. \& Lorenzi, H. 2008. Botânica sistemática: guia ilustrado para identificação das famílias de Fanerógamas nativas e exóticas no Brasil, baseado em APG II. Editora Plantarum, Nova Odessa. Pp. 704.

Stannard, B. L. 1995 (ed.). Flora do Pico das Almas: Chapada Diamantina-Bahia, Brazil. Royal Botanic Gardens, Kew, 853p.

Stradmann, M. T. S. 1997. Composição florística de um trecho da mata ciliar da Trilha do Bordãoe estudo quantitativo do estrato arbóreoarbustivo, Rio Ribeirão. Parque Nacional da Chapada Diamantina, Bahia, Brasil. Monografia de Graduação. Universidade Federal da Bahia, Salvador, 69p.

Stradmann, M. T. S. 2000. Composição florística da mata ciliar da foz do Rio Capivara e análise quantitativa do estrato arbustivo-arbóreo. Parque Nacional da Chapada Diamantina. Dissertação de Mestrado. Universidade Federal da Bahia, Salvador, 130p.

Veloso, H. P.; Rangel Filho, A. L. R. \& Lima, J. C. A. 1991. Classificação da vegetação brasileira, adaptada a um sistema universal. Fundação Instituto Brasileiro de Geografia e Estatística, Rio de Janeiro, 123p.

Walter, B. M. T. 1995. Distribuição especial das espécies perenes em uma mata de galeria inundável no Distrito Federal; florística e fitossociologia. Dissertação de Mestrado. Universidade de Brasília, Brasília, 150p. 